University of Wollongong

Research Online

Australian Institute for Innovative Materials -

Papers

Australian Institute for Innovative Materials

$1-1-2018$

Exploiting Intermolecular Interactions between Alkyl-Functionalized RedoxActive Molecule Pairs to Enhance Interfacial Electron Transfer

Inseong Cho

University of Wollongong, ic412@uowmail.edu.au

Mizuho Koshika

Shinshu University

Pawel W. Wagner

University of Wollongong, pawel@uow.edu.au

Nagatoshi Koumura

University of Tsukuba

Peter C. Innis

University of Wollongong, innis@uow.edu.au

See next page for additional authors

Follow this and additional works at: https://ro.uow.edu.au/aiimpapers

Part of the Engineering Commons, and the Physical Sciences and Mathematics Commons

Research Online is the open access institutional repository for the University of Wollongong. For further information contact the UOW Library: research-pubs@uow.edu.au 


\title{
Exploiting Intermolecular Interactions between Alkyl-Functionalized Redox-Active Molecule Pairs to Enhance Interfacial Electron Transfer
}

\author{
Abstract \\ The strategies to enhance electron transfer rates between redox-active, light-harvesting molecules \\ attached to semiconductor surfaces and redox mediators in solution by modifying molecular structure \\ are not fully investigated yet. Therefore, the design of molecules with controlled electron transfer rates \\ remains a challenge. The aims of this work are to quantify the effect of long alkyl chain substitution on \\ the electron transfer from cobalt(II/III) tris(2,2'-bipyridine) to organic molecules containing carbazole and \\ thiophene and to demonstrate that alkyl chains can be used to enhance electron transfer between donor- \\ acceptor pairs. To this end, we study the effect of using a combination of donor and acceptor molecules \\ with and without alkyl chains on electron transfer kinetics. Using transient absorption spectroscopy, we \\ show that when only the molecules or the mediators have long alkyl chains, electron transfer is slightly \\ blocked as expected. Counterintuitively, electron transfer is up to 13 times faster when long alkyl chains \\ are attached to both the redox-active molecules and the redox mediators. The faster electron transfer is \\ explained by an alkyl-alkyl chain interaction between the donor/acceptor, leading to the proximity \\ (trapping) of the redox mediators close to the $\pi$-conjugated backbone of the molecules. These results \\ suggest that intermolecular interactions can be used to enhance the electron transfer rates significantly \\ even with well-established insulating alkyl chains attached to molecules without changing the \\ electrochemical driving force.

\section{Disciplines} \\ Engineering | Physical Sciences and Mathematics

\section{Publication Details} \\ Cho, I., Koshika, M., Wagner, P., Koumura, N., Innis, P. C., Mori, S. \& Mozer, A. J. (2018). Exploiting \\ Intermolecular Interactions between Alkyl-Functionalized Redox-Active Molecule Pairs to Enhance \\ Interfacial Electron Transfer. Journal of the American Chemical Society, 140 (42), 13935-13944.
}

\section{Authors}

Inseong Cho, Mizuho Koshika, Pawel W. Wagner, Nagatoshi Koumura, Peter C. Innis, Shogo Mori, and Attila J. Mozer 


\title{
Exploiting intermolecular interactions between alkyl-functionalized redox active molecule pairs to enhance interfacial electron transfer
}

\author{
Inseong Cho, ${ }^{\dagger}$ Mizuho Koshika, ${ }^{\ddagger}$ Pawel Wagner, ${ }^{\dagger}$ Nagatoshi Koumura,,${ }^{\S}$ Peter C. Innis, ${ }^{\dagger}$ Shogo \\ Mori, ${ }^{\ddagger}$ and Attila J. Mozer*,† \\ tIntelligent Polymer Research Institute and ARC Centre of Excellence for Electromaterials Science, University of \\ Wollongong, New South Wales 2522, Australia \\ ‡Division of Chemistry and Materials, Faculty of Textile Science and Technology and Centre for Energy and Envi- \\ ronmental Science, Shinshu University, Ueda, Nagano 386-8567, Japan \\ \$Interdisciplinary Research Center for Catalytic Chemistry, National Institute of Advanced Industrial Science and \\ Technology (AIST), 1-1-1 Higashi Tsukuba, Ibaraki 203-8565, Japan
}

KEYWORDS Electron transfer, interfacial charge transfer, charge transfer kinetics, structural modification, redox active molecules, redox mediators, metal-ligand coordination complexes, cobalt bipyridine complexes, alkyl substitutions, transient absorption spectroscopy, intermolecular interaction, alkyl-alkyl chain interaction

\begin{abstract}
Strategies to enhance electron transfer rates between redox active, light harvesting molecules attached to semiconductor surfaces and redox mediators in solution by modifying molecular structure are not fully investigated yet. Therefore, the design of molecules with controlled electron transfer rates remains a challenge. The aims of this work are to quantify the effect of long alkyl chain substitution on the electron transfer from cobalt (II/III) tris(2,2' - bipyridine), $(\text { Cobpy })^{2+3}$ to organic molecules containing carbazole and thiophene and to demonstrate that alkyl chains can be used to enhance electron transfer between donor acceptor pairs. To this end, we study the effect of using a combination of donor and acceptor molecules with and without alkyl chains on electron transfer kinetics. Using transient absorption spectroscopy, we show that when only the molecules or the mediators have long alkyl chains, electron transfer is slightly blocked as expected. Counterintuitively, electron transfer is up to thirteen times faster when long alkyl chains are attached to both the redox active molecules and the redox mediators. The faster electron transfer is explained by an alkyl - alkyl chain interaction between the donor / acceptor leading to the proximity (trapping) of the redox mediators close to the $\pi$-conjugated backbone of the molecules. These results suggest that intermolecular interactions can be used to enhance the electron transfer rates significantly even with well-established insulating alkyl chains attached to molecules without changing the electrochemical driving force.
\end{abstract}

\section{INTRODUCTION}

The control of electron transfer between redox active molecules at charge transfer interfaces are fundamentally important in chemistry, physics, and biology. ${ }^{1-7}$ For example, the development of solar energy conversion technologies such as photoelectrosynthetic cells performing water splitting, $\mathrm{CO}_{2}$ or $\mathrm{N}_{2}$ reduction rely on efficient transfer of photo-generated charges from the light harvesting sites to the catalytic active sites where the dark reactions occur..$^{8-12}$ One of the main factors controlling the electron transfer rate is the electrochemical driving force $(\Delta G)$ for the reaction, i.e. the electrochemical energy difference between the electron donor and the acceptor pair. However, increasing the driving force by adjusting the chemical structure decreases the electrochemical energy stored in the products. Larger driving force leads to lower photon to chemical or electrochemical conversion efficiency. Therefore, to achieve high conversion efficiency, it is necessary to find mechanisms to enhance electron transfer rates besides driving force.

In 2016, Ogawa et al. showed that the nature of the donor group influenced the electron transfer kinetics between a series of organic molecules containing triphenylamine (TPA), carbazole or indolene donor units on $\mathrm{TiO}_{2}$ and the iodide / triiodide redox mediator in a solution. Although the driving force for electron transfer between the TPA containing molecules and the redox mediator was less, the electron transfer was enhanced by approximately a factor of seven at complete coverage of the semiconductor surface. This enhancement was attributed to the tridimensional nature of the TPA unit. It was argued that the redox mediator could not easily penetrate the closely packed acceptors (oxidized molecules) attached to the $\mathrm{TiO}_{2}$ surface. Since the hole is mostly delocalized along the $\pi$-conjugated segments of the molecules, electron transfer is restricted to 


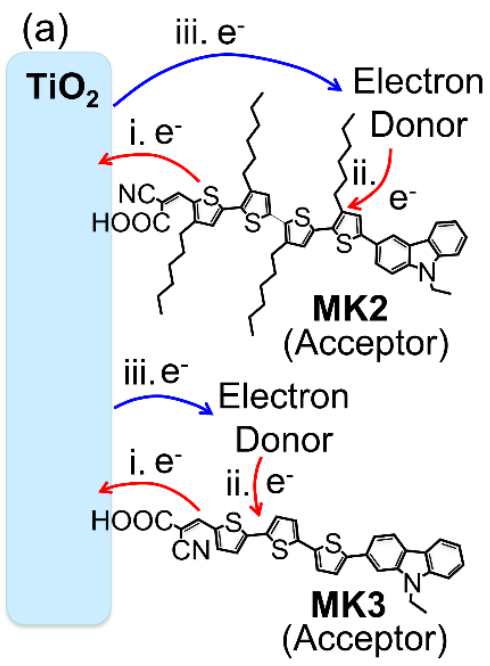

(b) $(\text { Cobpy })^{2+/ 3+}(\text { Cobpy-C1 })^{2+/ 3+}$

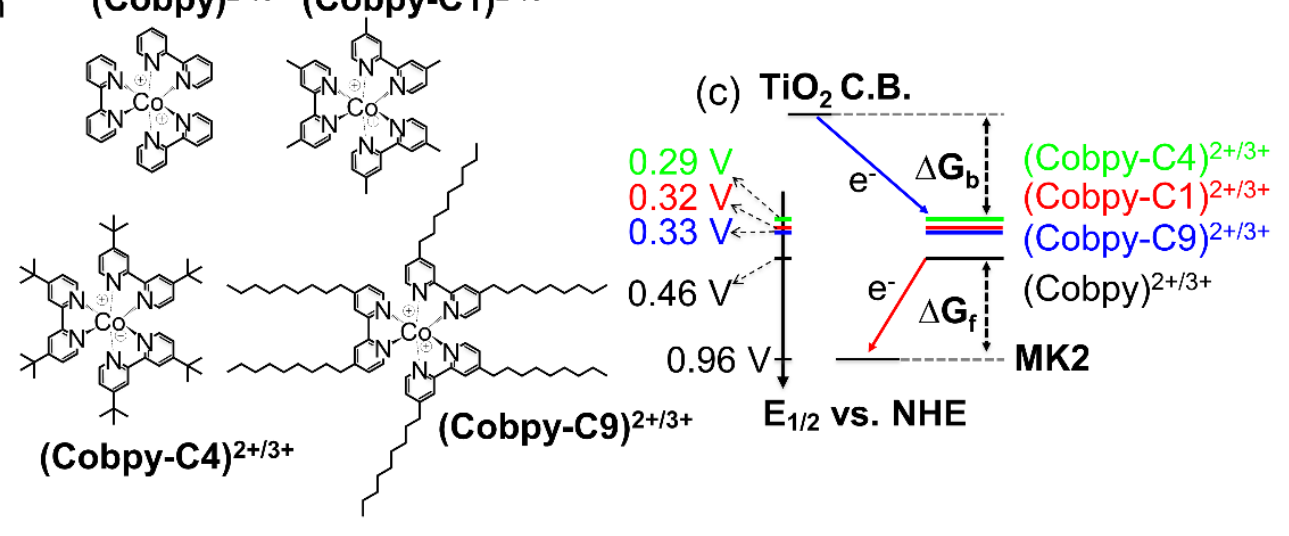

Figure 1. Chemical structures of $\mathrm{MK}_{2}$ and $\mathrm{MK}_{3}$ (a) and the redox mediators (b) studied in this work. Illustrations of forward (ii) and back (iii) electron transfer processes (a), and energy diagram constructed from redox potentials taken from Sapp et al. (cobalt complexes) ${ }^{13}$ and Wang et al. (MK2 $)^{14}$. Driving force for forward $\left(\Delta G_{f}\right)$ and back $\left(\Delta G_{b}\right)$ electron transfer.

the end units of the molecules exposed towards the electrolyte. The three-dimensional TPA unit provides a larger cross-sectional area for electron transfer at the molecular / electrolyte interface as compared to the more planar carbazole or indolene units. On the other hand, when the concentration of the molecules on the surface was reduced, the electron transfer rate was generally faster and followed the order of increasing electrochemical driving force among the molecules. This suggests that the effect of molecular structure on electron transfer could be different in the case of surface-bound molecules compared to homogeneous electron transfer reactions in solution. In the case of a loosely packed acceptor layer, the $\pi$-conjugated backbone of the molecules was more accessible to the redox mediator leading to faster electron transfer, but one should design molecules to have both easy accessibility and high adsorption density simultaneously. In 2016, this group of authors showed that attaching TPA-carbazole units to porphyrin molecules on $\mathrm{TiO}_{2}$ enhanced the electron transfer rate by up to five times at the same driving force, which was attributed to the more exposed $\pi$-conjugated orbitals of the TPA - carbazole unit towards the redox electrolyte. ${ }^{15}$ The above studies show that the design of the molecular structure to enable faster electron transfer is different for surface adsorbed molecules at high packing density from that of free molecules in solution. ${ }^{16}$

Insulating alkyl chains are often attached to redox active molecules used in photo-electrochemical cells to slow down unwanted back electron transfer. Back electron transfer kinetics refers to the reduction of the oxidized form of the redox mediator by the photogenerated electrons in the $\mathrm{TiO}_{2}$ and is often characterized by measuring electron lifetime. Electron lifetime at the same charge density increased approximately by an order of magnitude at matched electron density when hexyl chains were attached to carbazole containing organic molecules, see molecular structures of $\mathrm{MK}_{2}$ and $\mathrm{MK}_{3}$ in Figure $1 \mathbf{1}^{17}{ }^{17}$ The slower back electron transfer between the electron injected from the photo-excited molecule into the $\mathrm{TiO}_{2}$ particle and the electron donor in a solution is explained by the "blocking effect" of the alkyl chains at the semiconductor / electrolyte interface, indicated by the blue arrow in Figure 1a. A highly packed acceptor layer with less free space between them prevents the donor's approach to the semiconductor surface, hence increases the distance over which back electron transfer operates. Using sterically hindered molecules can also effectively increase the lifetime of photoinjected electrons in the semiconductor by increasing the distance between the semiconductor surface and redox species in the electrolyte..$^{18-20}$

Alkyl chains can be also attached to redox mediators instead. In 2002, Sapp et al. explored the feasibility of aryl or alkyl-substituted cobalt polypyridyl complexes as redox mediators for photoelectrochemical solar cells. ${ }^{13} 14$ different cobalt complexes were tested in dye-sensitized solar cells looking for desirable structural and thermodynamic motifs. The interesting observation was an increasing open circuit voltage as the number of carbon atoms in the attached aryl or alkyl chains increased. The short circuit current peaked at four carbon atoms (tert-butyl). While back electron transfer or forward electron transfer rates were not measured, the findings were explained by blocking back electron transfer in the better performing devices.

For the case of the combination of both acceptor and donor molecules with and without alkyl chains, the effect of alkyl chains and driving force has not been clearly distinguished yet. Feldt et al. showed in 2010 that back electron transfer was retarded by attaching insulating tert-butyl chains to cobalt polypyridyl redox molecules. ${ }^{19}$ The longer 
electron lifetime between unsubstituted and methyl substituted mediators was explained by the smaller driving force for back electron transfer, whereas the steric effect of tert-butyl chains contributed to a further increase due to longer distance between the $\mathrm{TiO}_{2}$ electron and the oxidized redox mediator. They also showed that when a surface-bound molecule with alkoxy substitution was used (D35, see Figure S1), the effect of alkyl chain substitution on the redox mediator was less noticeable. It is noted that tert-butyl pyridine (tBP), a common additive used to enhance photovoltaic device performance, was also added to the electrolyte, which itself has an effect on electron lifetime and may influence the molecule / redox mediator interaction at the surface. Similarly to the study by Feldt et. al., increased electron lifetime using tert-butyl substituted $(\text { Cobpy })^{2+/ 3+}$ redox mediator in combination with large carbazole containing molecules was reported by Ohta et al. ${ }^{18}$ In 2011, Feldt et al. measured the forward electron transfer rate (process ii in Figure 1a) between the oxidized acceptor molecules $\left(D_{35}\right)$ and the cobalt mediators in the presence of tBP additive using transient absorption spectroscopy. ${ }^{21}$ At the same driving force, the electron transfer from tertbutyl substituted cobalt tris(bipyridine) was approximately $35 \%$ slower than methyl substituted one, which was explained by the blocking effect of the tert-butyl chain.

Questions arise, to what extent long insulating alkyl chains attached to redox mediators can slow down the forward electron transfer rate, both in the presence and in the absence of alkyl chains on the electron acceptor molecules? Based on a simple model of distance dependent electron transfer rate ${ }^{22}$ controlled by the length of the alkyl chains, the presence of alkyl chains on both donor and acceptor molecules could further decrease the electron transfer due to even larger distance between the two molecules. However, specific interactions between the donor and acceptor at the charge transfer interface may complicate this simple model.

To answer the above question, we have synthesized four cobalt tris(bipyridine) redox mediators with a series of insulating alkyl chains, see Figure $\mathbf{1 b}$, cobalt(II/III) tris $\left(4,4^{\prime}-\right.$ dimetyl-2,2'-bipyridine), (Cobpy-C 1$)^{2+/ 3+}, \quad$ cobalt(II/III) tris (4,4'-di-tert-butyl-2,2'-bipyridine), $\quad(\text { Cobpy- } 44)^{2+/ 3+}$, and cobalt(II/III) tris(4,4'-dinonyl-2,2'-bipyridine), (Cobpy-C9 $)^{2+/ 3+}$, and compared to the unsubstituted complex, cobalt (II/III) tris(2,2'-bipyridine), (Cobpy) ${ }^{2+/ 3^{+}}$. The redox potential of these compounds have already been reported by Sapp et al. ${ }^{13}$ An energy level diagram is constructed as shown in Figure 1c. Due to the negligible differences in the redox potential of the methyl and nonyl substituted cobalt complexes, the effect of long alkyl chain substitution on electron transfer at negligible change in the driving force for both forward electron transfer $\left(\Delta G_{f}\right)$ and back electron transfer $\left(\Delta \mathrm{G}_{\mathrm{b}}\right)$ can be studied.

We choose two well studied organic molecules MK2 and $\mathrm{MK}_{3},{ }^{14}$ with hexyl chains (2-cyano-3-[5"'-(9-ethyl-9H-carbazol-3-yl)-3',3",3"',4-tetra-n-hexyl-[2,2',5,2",5",2"']-quaterthiophenyl-5-yl]acrylic acid, MK2) and without alkyl chains (2-cyano-3-[5"-(9-ethyl-9H-carbazol-3-yl)$\left[2,2^{\prime}, 5^{\prime}, 2^{\prime \prime}\right]$ terthiophenyl-5-yl]acrylic acid, Mk3 to pair with the cobalt complexes.

First, since there is some discrepancy between the reported redox potential values for the cobalt complexes in the literature, we measured the redox potentials using cycling voltammetry (Figure $\mathbf{2}$ and S2). Nanosecond and microsecond transient absorption (TA) measurements were performed to determine forward electron transfer rates between oxidized $\mathrm{MK}_{2} / \mathrm{MK}_{3}$ molecules attached to a $\mathrm{TiO}_{2}$ surface and the redox mediators dissolved in the electrolyte at two different concentrations (Figures 6 to 8). The results shown here did not contain $\mathrm{tBP}$. To allow comparison with literature and to establish our hypothesis for molecular interactions, we also investigated the effect of alkyl chain substitution on the back electron transfer reaction using small amplitude photovoltage and photocurrent decay measurements combined with charge extraction (Figures ${ }_{3}, S_{4}$ and $\left.S_{5}\right){ }^{23}$

\section{RESULTS AND DISCUSSION}

Redox potentials and calculation of driving force for electron transfer. The redox potentials of all six compounds investigated here has been already reported..$^{13-14,24-}$ ${ }_{25}$ Due to differences in experimental conditions (solvent, supporting electrolyte, working and references electrodes) the redox potentials were measured at the same conditions using cycling voltammetry to estimate the driving force for forward and backward electron transfer.
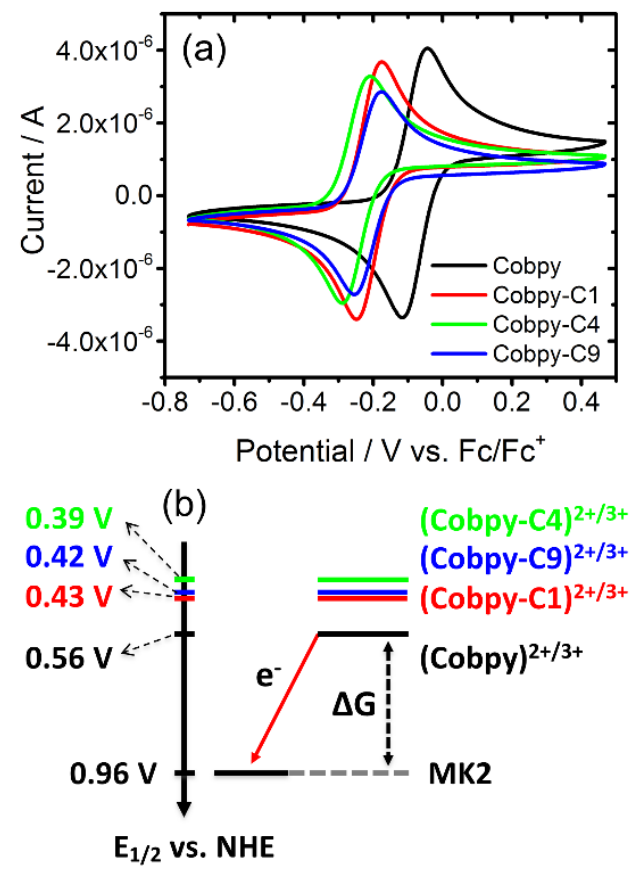

Figure 2. Current versus potential curves measured by cyclic voltammetry of the cobalt complexes (a) and energy level diagram constructed using the measured $E_{1 / 2}$ values (b). 
Table 1. Half-wave potentials ( $E_{1 / 2}$, versus vacuum) of molecules used in this work and the driving forces for forward (process ii in Figure 1a) electron transfer $\left(-\Delta \mathbf{G}_{\mathrm{f}}\right)$ and back (process iii in Figure 1a) electron transfer $\left(-\Delta \mathbf{G}_{\mathrm{b}}\right)$.

\begin{tabular}{|c|c|c|c|c|}
\hline Compound & $\mathrm{E}_{1 / 2}\left(\mathrm{~V}\right.$ vs. $\left.\mathrm{Fc} / \mathrm{Fc}^{+}\right)$ & $\mathrm{E}_{1 / 2}(\mathrm{eV})$ & $-\Delta \mathrm{G}_{\mathrm{f}}(\mathrm{eV})$ & $-\Delta \mathrm{G}_{\mathrm{b}}(\mathrm{eV})$ \\
\hline $\mathrm{MK2}$ & 0.322 & -5.422 & - & - \\
\hline$(\text { Cobpy })^{2+/ 3+}$ & -0.079 & -5.022 & 0.401 & 0.862 \\
\hline$(\text { Cobpy-C1 })^{2+/ 3+}$ & -0.211 & -4.890 & 0.533 & 0.730 \\
\hline$\left(\text { Cobpy }-C_{4}\right)^{2+/ 3+}$ & -0.249 & -4.851 & 0.571 & 0.691 \\
\hline$(\text { Cobpy-C9 })^{2+/ 3+}$ & -0.216 & -4.884 & 0.538 & 0.724 \\
\hline
\end{tabular}

The redox potential of $\mathrm{MK}_{2}$ anchored to a $\mathrm{TiO}_{2}$ surface measured using cycling voltammetry $(\mathrm{CV})$ is shown in Table 1, while the CV is displayed in Figure $\mathbf{S}_{\mathbf{3}}$. The CV of $\mathrm{MK}_{2}$ on $\mathrm{TiO}_{2}$ showed two reversible peaks with the halfwave potential of the first peak $\mathrm{E}_{1 / 2}=0.322 \mathrm{Vvs}$. $\mathrm{Fc} / \mathrm{Fc}^{+}(\mathrm{Ta}-$ ble 1 and Figure $\left.S_{3}\right)$. Wang et al. have reported the redox potential of the first oxidation peak (o.96 V versus NHE) which is exactly the same value we obtained. The second peak is attributed to the oxidation of $\mathrm{MK2}^{+}$to the di-cation $\mathrm{MK2}^{2+} .25$ The $\mathrm{E}_{1 / 2}$ value of $\mathrm{MK}_{3}$ was reported to be $0.030 \mathrm{~V}$ more positive compared to $\mathrm{MK}_{2}$, however the $\mathrm{CVs}$ were not shown. ${ }^{14}$ Several attempts to measure $\mathrm{E}_{1 / 2}$ of $\mathrm{MK}_{3}$ adsorbed on $\mathrm{TiO}_{2}$ by cyclic voltammetry was not successful, yielding non-reversible reduction peaks. The as prepared $\mathrm{MK}_{3}$ adsorbed $\mathrm{TiO}_{2}$ film had an orange color. After an oxidation cycle to up to $1 \mathrm{~V}$ versus $\mathrm{Ag} / \mathrm{AgNO}_{3}$ reference, the sample became transparent suggesting $\mathrm{MK}_{3}$ was no longer attached to the $\mathrm{TiO}_{2}$ surface. When using thicker $\mathrm{TiO}_{2}$ films, the film became darker suggesting non reversibility of the $\mathrm{MK}_{3}$ electrochemistry on $\mathrm{TiO}_{2}$, making the determination of $\mathrm{E}_{1 / 2}$ unreliable.

The CVs of the four cobalt complex redox mediators (platinum working and counter electrodes, o.o1 M $\mathrm{Ag} / \mathrm{AgNO}_{3}$ reference) are shown in Figure $2 \mathbf{a}$ and $\mathbf{S}_{2}$, while the calculated $\mathrm{E}_{1 / 2}$ versus $\mathrm{Fc} / \mathrm{Fc}^{+}$and versus vacuum values are displayed in Table 1 and Table S1. Sapp et al. measured these four redox mediators using a gold working electrode, a platinum flag auxiliary electrode, and a $\mathrm{Ag} / \mathrm{Ag}^{+}$reference electrode (o.o1 $\mathrm{M} \mathrm{AgNO}_{3}$ in dimethyl sulfoxide) (See Figure 1c). ${ }^{33}$ Similarly to the results of Sapp et al., the redox potential of the alkyl substituted cobalt complexes shifted towards more negative potentials by approximately 130 $\mathrm{mV}$, which is explained by the increased electron density on the $\mathrm{Co}^{2+/ 3+}$ due to the presence of alkyl chains. The redox potentials of the methyl and nonyl substituted complexes were very similar (within $6 \mathrm{mV}$ ) of each other, while the tert-butyl substituted cobalt complex showed a negative shift by $39 \mathrm{mV}$. These results are generally consistent with the results of Sapp et al., but with a major difference in the absolute values of the redox potentials by approximately $100 \mathrm{mV}$, compare Figure $\mathbf{1 c}$ and Figure $\mathbf{2 b}$. The possible origin of this difference could arise from the use of different solvent and supporting electrolyte. The reference electrode potential used in the work by Sapp et al. was $0.47 \mathrm{~V}$ versus SHE. The reference electrode used in this work was calibrated using the $\mathrm{Fc} / \mathrm{Fc}^{+}$couple and converted to NHE using $0.64 \mathrm{~V}$ following the work of Cardona et al. ${ }^{26}$

Feldt et al. in $2010^{19}$ and $2011^{21}$ reported the redox potentials of three of the cobalt mediators, showing $0.56 \mathrm{~V}$ versus NHE for the unsubstituted (Cobpy) $)^{2+/ 3^{+}}$and same (within two digits) potentials for methyl and tert-butyl substituted compounds (o.43 V). Similar to the results shown here, they used a platinum working electrode and a $\mathrm{Ag} / \mathrm{Ag}^{+}$reference electrode composed of o.o1 $\mathrm{M} \mathrm{AgNO}_{3}$, calibrated versus $\mathrm{Fc} / \mathrm{Fc}^{+}$. The value of $\mathrm{Fc} / \mathrm{Fc}^{+}$to NHE scale was not reported. Our result matches the values of the redox potentials reported by Feldt et al. However, the values of the alkyl substituted cobalt complexes were different. Most notably, the redox potential of the tert-butyl substituted compound showed a positive shift by $39 \mathrm{mV}$ compared to the methyl substituted as already mentioned above, while it was found to be the same as the redox potential of the methyl substituted one in the work of Feldt et al. The standard deviation of the $\mathrm{E}_{1 / 2}$ was determined by repeating the CV scans four times and in addition, measuring a second, independently prepared sample (Figure $\mathbf{S}_{2}$ ). The standard variation was in the range of 1 to $3 \mathrm{mV}$, which is similar to the voltage resolution of the CV experiment (1 $\mathrm{mV})$. To conclude, the redox potentials of the four compounds were measured under the same experimental conditions. The methyl and nonyl substituted mediators had nearly the same redox potentials (within $6 \mathrm{mV}$ ), hence nearly identical driving force for electron transfer $0.53 \mathrm{eV}$ when paired with MK2. Due to the shift of its redox potential, the tert-butyl substituted mediator provides a $40 \mathrm{mV}$ larger driving force ( $0.57 \mathrm{eV}$ with $\mathrm{MK}_{2}$ ) while the unsubstituted (Cobpy) $)^{2+3+}$ provides a $130 \mathrm{mV}$ less driving force (o.40 $\mathrm{eV}$ with MK2). Therefore, the effect of long alkyl substitution on forward and backward electron transfer can be readily compared using (Cobpy- $\left.\mathrm{C}_{1}\right)^{2+/ 3+}$ and (Cobpy$\left.\mathrm{C}_{9}\right)^{2+/ 3^{+}}$, see the energy level diagram with the values measured in this work (Figure $\mathbf{2 b}$ ).

Back electron transfer kinetics (process iii in Figure 1a). Upon photo-excitation of $\mathrm{MK}_{2}$-adsorbed $\mathrm{TiO}_{2}$ films using a $635 \mathrm{~nm}$ laser, electrons are injected from the photoexcited $\mathrm{MK}_{2}$ molecules into the conduction band of the $\mathrm{TiO}_{2}$. In case of fast reduction of the oxidized $\mathrm{MK}_{2}$ molecules by the reduced form of the mediator (process ii in Figure 1a), the injected electrons recombine with the oxidized form of the redox mediator (process iii). The rate of 

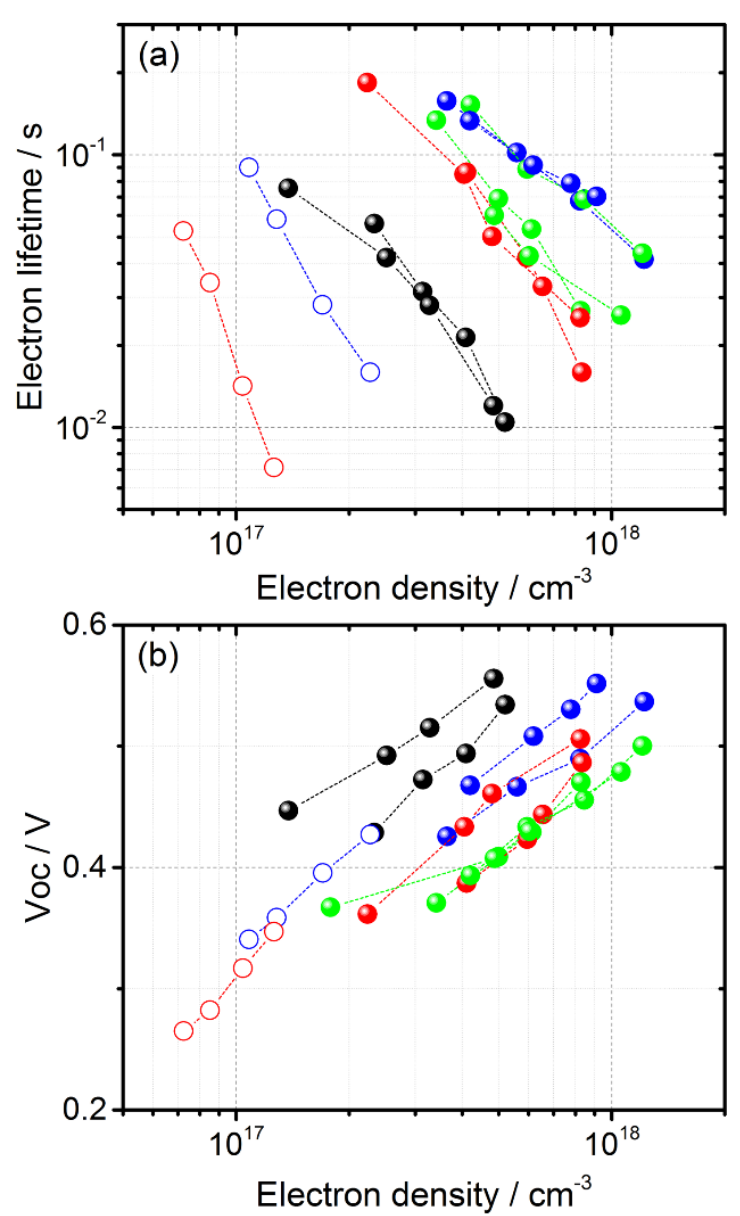

Figure 3. Electron lifetime $(\tau)$ versus charge density measured for $\mathrm{MK}_{2}-\mathrm{TiO}_{2}$ samples (a) and open circuit voltage $\left(\mathrm{V}_{\mathrm{oc}}\right)$ versus charge density (b) using (Cobpy) $2+/ 3^{+}$(black), (Cobpy$\left.\mathrm{C}_{1}\right)^{2+13+}$ (red), (Cobpy-C4) $)^{2+13+}$ (green), and (Cobpy-C9) $)^{2+/ 3+}$ (blue) electrolytes. Empty circles indicate lifetime values determined for $\mathrm{MK}_{2}-\mathrm{TiO}_{2}$ samples using (Cobpy-C $\left.)_{1}\right)^{2+/ 3+}$ (red) and (Cobpy-C9) $)^{2+/ 3+}$ (blue) electrolytes with lower (17\%) MK2 surface coverage.

this reaction depends on the electron density in the $\mathrm{TiO}_{2}{ }^{21}$ the concentration of the oxidized redox mediators near the $\mathrm{TiO}_{2}$ interface, ${ }^{23}$ the distance between the $\mathrm{TiO}_{2}$ electron and the redox mediators, and the driving force. ${ }^{19,23}$ Electron lifetime determined at the same charge density and at the same driving force can be used to quantitatively determine the effect of alkyl chain substitution (nonyl versus methyl), providing insights into the nature of intermolecular interactions at the semiconductor / molecular / electrolyte interface.

Electron lifetime $(\tau)$ obtained by voltage decay measurements of $\mathrm{MK}_{2}$-adsorbed $\mathrm{TiO}_{2}\left(\mathrm{MK}_{2}-\mathrm{TiO}_{2}\right)$ samples (at least two identically prepared samples for each condition) are shown in Figure 3a, while the $\mathrm{V}_{\text {oc }}$ versus charge density plots obtained by charge extraction are shown in Figure 3b. Samples containing the methyl substituted redox mediator (o.2 M / $0.02 \mathrm{M}$, (Cobpy-C1 $)^{2+/ 3^{+}}$) showed five times longer lifetime at the same charge density compared to the unsubstituted (Cobpy) $)^{2+/ 3+}$ (see Table 2). Samples prepared with the nonyl substituted mediator showed an additional two-fold longer lifetime. The lifetime measurements of the samples containing the tert-butyl substituted redox mediator were somewhat less reproducible. Therefore the measurements were repeated several times and the results for three samples are shown. On average, the lifetime using tert-butyl substituted mediator was $40 \%$ longer compared to the methyl substituted (Cobpy-C1 $)^{2+/ 3+}$ at the same charge density.

The open-circuit voltage $\left(\mathrm{V}_{\mathrm{oc}}\right)$ of these samples is given by the difference in the Fermi level in the $\mathrm{TiO}_{2}$ (charge density dependent) and the redox potential of the electrolyte at the platinum electrode (dependent on the activity of the redox mediator). ${ }^{19}$ Comparing to the samples containing unsubstituted (Cobpy) ${ }^{2+/ 3+}$, the $\mathrm{V}_{\text {oc }}$ shifted on average by approximately $100 \mathrm{mV}$ at the same charge density using the methyl substituted cobalt complex, consistent with the negative shift in its redox potential (Table 2). Based on the $E_{1 / 2}$ values, a larger shift of approximately $130 \mathrm{mV}$ is expected (Table 1). Note that the reproducibility of the charge extraction measurements in the absence of tBP is around $30 \mathrm{mV}$, so the difference between the expected and measured $V_{\text {oc }}$ change in devices at least partially can be attributed to a measurement error. Any remaining difference can be attributed to i) the shift of the $\mathrm{TiO}_{2}$ conduction band bottom edge potential $\left(E_{c b}\right)$ or ii) a shift of the redox potential at the Pt counter electrode. $\mathrm{E}_{\mathrm{cb}}$ is determined by the adsorbed electrolyte cation density $\left(\mathrm{Li}^{+}\right.$in our case) on the $\mathrm{TiO}_{2}$ surface, which also influences the electron diffusion coefficient. ${ }^{23}$ The similar diffusion coefficients at the same short circuit current density (Figure $\mathbf{S}_{4}$ ) suggest similar absorbed cation density between samples. However, the diffusion coefficient can be less sensitive to any changes in the adsorbed cation density, so any small shift in $E_{c b}$ between the samples cannot be ruled out. It is noted that both electron density and diffusion coefficient were measured shortly after cell fabrication within 20 minutes. Within this time frame, the $\mathrm{E}_{\mathrm{cb}}$ can shift depending on how easily the cations can access the $\mathrm{TiO}_{2}$ surface. Importantly, the measured $\mathrm{V}_{\text {oc }}$ on average was $30 \mathrm{mV}$ lower when the tert-butyl substituted cobalt mediator was used, which is consistent with the negative shift of $E_{1 / 2}$ compared to (Cobpy-C 1$)^{2+/ 3+}$, supporting the accuracy of the CV measurements.

The five-fold longer $\tau$ for $\left(\text { Cobpy- } \mathrm{C}_{1}\right)^{2+/ 3^{+}}$can be attributed to the reduced driving force for electron transfer $\left(\Delta \mathrm{G}_{\mathrm{b}}\right)$, similarly to the explanation of Feldt et al. However, the two-fold longer $\tau$ using the nonyl substituted redox mediator (Cobpy-C9) $)^{2+/ 3+}$ compared to the methyl substituted one cannot be explained by a driving force effect. It is therefore suggested that the longer alkyl chain leads to an increased distance between the $\mathrm{TiO}_{2}$ surface and the $(\text { Cobpy-C9 })^{3+}$ in the electrolyte, leading to longer lifetime. 


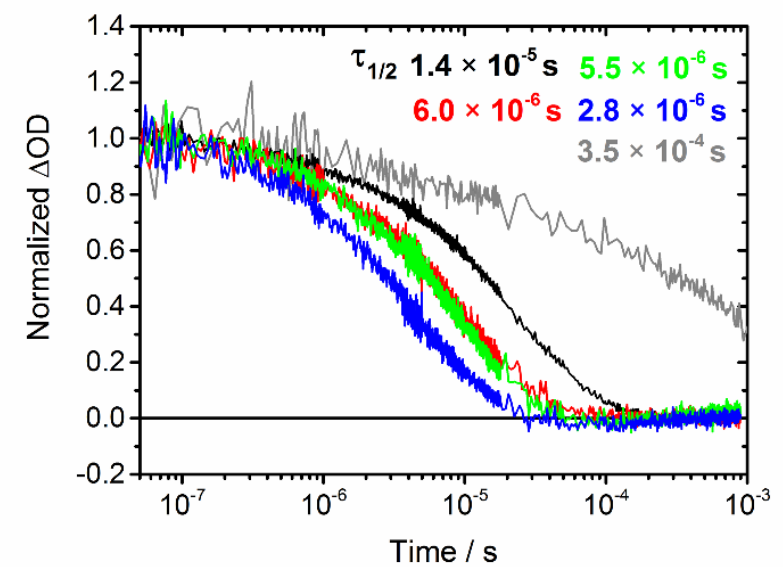

Figure 4. Normalized $\Delta \mathrm{OD}$ decays (at $800 \mathrm{~nm}$ ) of $\mathrm{MK}_{2}-\mathrm{TiO}_{2}$ samples prepared using $0.2 \mathrm{M} \mathrm{Co}^{2+}, 0.02 \mathrm{M} \mathrm{Co}^{3+}$ in $0.2 \mathrm{M}$ $\mathrm{LiClO}_{4}$ acetonitrile. (Cobpy) ${ }^{2+/ 3+}$ (black), (Cobpy-C1 $)^{2+/ 3+}$ (red), $(\text { Cobpy-C } 4)^{2+/ 3+}$ (green), (Cobpy-C 9$)^{2+/ 3+}$ (blue), and inert electrolyte (grey).

The lifetime data does not clearly confirm the blocking effect of the tert-butyl substituted redox mediator in combination with $\mathrm{MK}_{2}$, as both the molecular structure and redox potential changes. The approximately $40 \%$ longer lifetime is somewhat longer than expected based on the effect of driving force alone, implying a moderate blocking effect in the case of the tert-butyl substitution. We note that the larger variation in lifetime in Figure za could suggest a time-dependent and generally slower change at the $\mathrm{TiO}_{2} /$ electrolyte interface. Depending on whether linear or branched alkyl chain is used, the rate of change in the concentration of the redox mediator near the $\mathrm{TiO}_{2}$ interface can be sample dependent, causing a larger variation of the measured $\mathrm{V}_{\text {oc }}$ values in the case of the tert-butyl substituted cobalt complex mediator.

The results are consistent with the literature confirming moderate blocking effect of a branched or long alkyl chain substitution on the cobalt complex mediator. Each $\mathrm{MK}_{2}$ molecule contains four hexyl side chains therefore the benefit of alkyl chain substitution on the redox mediator may not provide significant additional blocking effect as noted by Feldt et al. in the case of their D 35 compound. Reducing the surface coverage of the molecular layer on the $\mathrm{TiO}_{2}$ surface is expected to lead to a more open space structure, creating a more accessible $\mathrm{TiO}_{2}$ surface for the redox mediator, hence shorter lifetime. Thus, reducing a surface coverage is an effective method to diminish the blocking effect of the molecular layer itself, enabling to amplify the blocking effect of alkyl chain substitution on the redox mediator itself. Figure 3 shows that at reduced MK2 coverage (17\% of full coverage, see experimental), the electron lifetime became shorter by over an order of magnitude. The decrease was less when the nonyl substituted cobalt mediator was used. The difference between using methyl and nonyl substituted mediators has increased compared to the full coverage MK2 films to almost an order of magnitude. The results show that the blocking effect of the nonyl chain is indeed more significant when the molecular layer on the surface is less compact. An explanation consistent with the literature is the increased distance between the redox mediator and the $\mathrm{TiO}_{2}$ surface. This increased distance can be the effect of longer alkyl chain (nonyl). However, this explanation is not consistent with the measurements of forward and back electron transfer rates involving the $\mathrm{MK}_{3}$ molecule as detailed in the following section.

Forward electron transfer kinetics (process ii in Figure 1a). The nonyl and tert-butyl moiety can block the back electron transfer between the $\mathrm{TiO}_{2}$ and the redox mediator, but does it influence the (forward) electron transfer between the redox mediator and the molecule on the surface (process ii in Figure 1a)?

Figure 4 shows the normalized change in the optical density $(\Delta \mathrm{OD})$ of oxidized $\mathrm{MK}_{2}$ molecules on the $\mathrm{TiO}_{2}$ surface in the absence (grey line) and presence of redox mediators (unsubstituted (black), methyl (red), tert-butyl (green), and nonyl (blue)) using a $0.2 \mathrm{M} \mathrm{LiClO}_{4}$ acetonitrile electrolyte. The $\triangle \mathrm{OD}$ was measured using transient absorption spectroscopy. The oxidized MK2 molecules were generated by electron injection from the MK2 photoexcited states into the $\mathrm{TiO}_{2}$. The rate of decay of the $\Delta \mathrm{OD}$ curves is directly related to the time dependent concentration of the $\mathrm{MK2}_{2}{ }^{+}$on the $\mathrm{TiO}_{2}$ surface. The faster decay in the presence of the redox mediators indicates faster electron transfer from the redox mediators to $\mathrm{MK2}^{+}$. Contrary to expectations and the back electron transfer measurements, the fastest decays were measured for the nonyl-substituted redox mediator. The driving force for forward electron transfer $\left(\Delta G_{f}\right.$, Table 1$)$ cannot explain the difference between the methyl and nonyl substituted mediators as they have nearly the same redox potentials and same $V_{\text {oc }}$ at

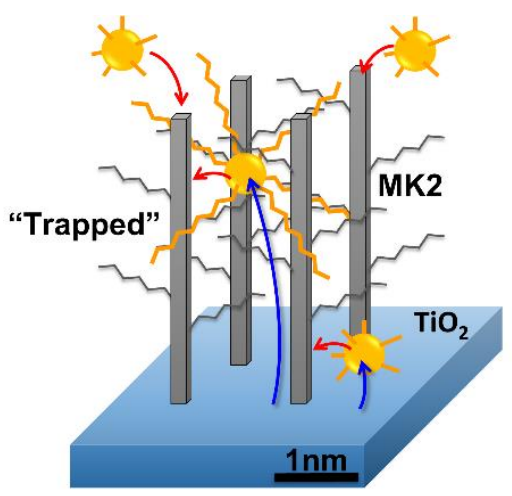

Figure 5. Schematic illustration of electron transfer at the $\mathrm{MK}_{2}-\mathrm{TiO}_{2} /$ electrolyte interface. Blue arrows indicate back electron transfer from the $\mathrm{TiO}_{2}$ to the oxidized mediator, while the red arrows indicate forward electron transfer from the reduced mediator to the oxidized MK2 molecules. 
Table 2. Averaged values of electron lifetime $(\tau)$ and open-circuit voltage $\left(V_{o c}\right)$ at the same charge density $(5.0 \times$ $\left.10^{17} \mathrm{~cm}^{-3}\right)$, and electron forward transfer rate $\left(k_{\mathrm{f}, 1 / 2}=1 / \tau_{1 / 2}\right)$ and rate constant $\left(K_{\mathrm{f}, 1 / 2}\right)$.

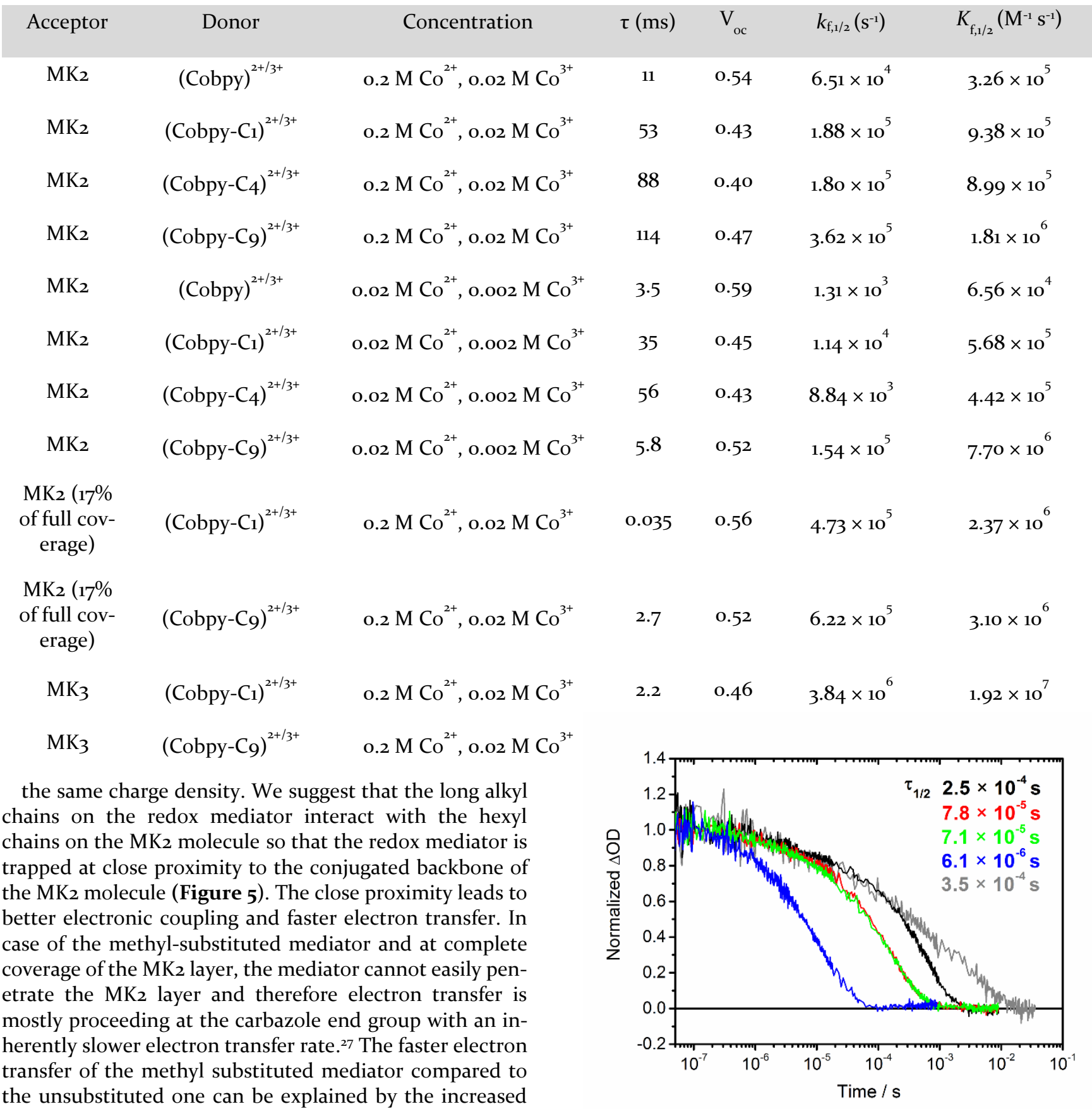

driving force for the forward electron transfer $\left(\Delta \mathrm{G}_{\mathrm{f}}\right)$. Based on the increased driving force using the tert-butyl substituted mediator, the kinetics is expected to be faster than the methyl-substituted one. The nearly identical kinetics suggest a slight blocking effect by the tert-butyl chain, canceling out the effect of driving force increase. The slight blocking effect for the tert-butyl substituted mediator for the forward electron transfer is consistent with the conclusion of Feldt et al.

Figure 6. Normalized $\Delta \mathrm{OD}$ decays (at $800 \mathrm{~nm}$ ) of $\mathrm{MK}_{2}-\mathrm{TiO}_{2}$ samples prepared using $0.02 \mathrm{M} \mathrm{Co}^{2+}, 0.002 \mathrm{M} \mathrm{Co}^{3+}$ in $0.2 \mathrm{M}$ $\mathrm{LiClO}_{4}$ acetonitrile. (Cobpy) ${ }^{2+/ 3+}$ (black), (Cobpy-C1 $)^{2+/ 3+}$ (red), $(\text { Cobpy-C } 4)^{2+/ 3+}$ (green), and (Cobpy-C9) $)^{2+/ 3+}$ (blue), and inert electrolyte (grey).

The transient absorption decays have been quantified using i) signal half decay times ( $\tau_{1 / 2}$, Table 2$)$ and ii) by fitting the decay curves to a stretched exponential function (Table S2). ${ }^{28}$ While these two methods lead to the same 
conclusion, we prefer to use the $\tau_{1 / 2}$ for further discussion since the stretched exponential function has more parameters. For completeness and for the benefit of interested readers, we provided the fitted parameters using a stretched exponential function (see SI and Table $\mathbf{S}_{3}$ ). For simplicity, we discuss the results using $\tau_{1 / 2}$.

The faster electron transfer measured for the nonyl substituted redox mediator in Figure $\mathbf{4}$ is important for a number of reasons. Firstly, electron transfer is enhanced at the same driving force. Secondly, intermolecular interactions such as the proposed alkyl - alkyl interaction are generally not considered when designing redox mediator / molecule pairs for charge transfer interfaces. Insulating alkyl chains can be barriers to electron transfer, however in this case the electron transfer was enhanced.

Testing the hypothesis of alkyl-alkyl interaction: Low electrolyte concentration, lower coverage of $\mathrm{MK}_{2}$ on $\mathrm{TiO}_{2}$, and the case of molecule without alkyl side chains $\left(\mathrm{MK}_{3}\right)$. To test our hypothesis, electron transfer rates were measured using a 10 -fold diluted electrolyte with the same amount of supporting electrolyte (0.2 M $\mathrm{LiClO}_{4}$ ). It is expected that at diluted bulk redox mediator concentrations, the enhancement due to the effect of alkyl - alkyl interaction at the surface should be larger compared to the case of high bulk redox concentration. This is because we expect that the enhancement in redox concentration at the charge transfer interface scales with the amount of surface bound molecules, however, it should not be dependent on the bulk redox concentration itself. Therefore, at higher bulk redox concentrations, the enhancement at the surface is masked. If other factors, such as change in the reorganization energy using the longer nonyl chain was responsible, the concentration should play no role and the rate should scale with the concentration. ${ }^{29}$ Figure 6 shows $\Delta \mathrm{OD}$ decays measured using transient absorption when

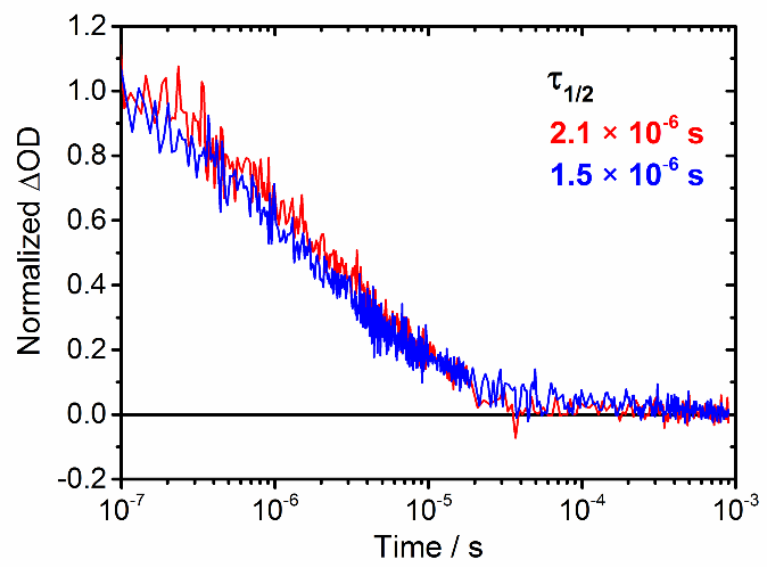

Figure 7. Normalized $\Delta \mathrm{OD}$ decays (at $800 \mathrm{~nm}$ ) of $\mathrm{MK}_{2}-\mathrm{TiO}_{2}$ (17\% of full coverage) samples prepared with $0.2 \mathrm{M} \mathrm{Co}^{2+}, 0.02$ $\mathrm{M} \mathrm{Co}^{3+}$ in $0.2 \mathrm{M} \mathrm{LiClO}_{4}$ acetonitrile. (Cobpy-C1) ${ }^{2+/ 3+}$ (red) and (Cobpy-C9) ${ }^{2+/ 3+}$ (blue).

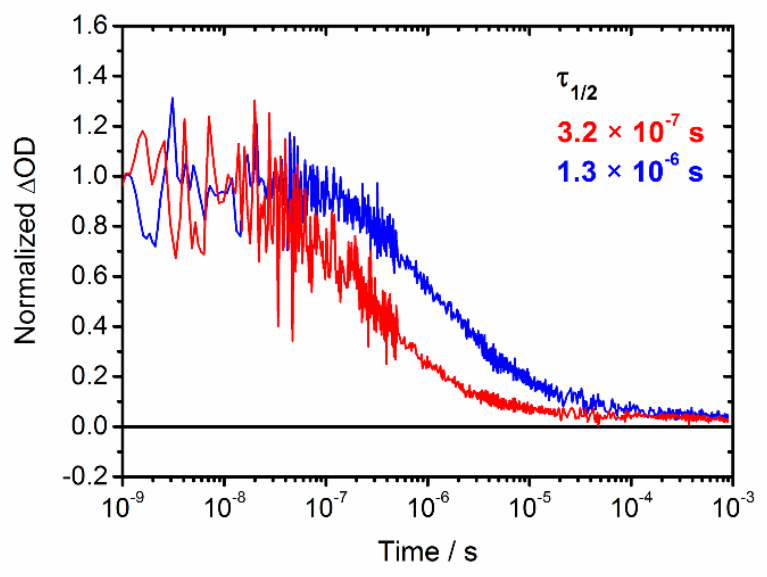

Figure 8. Normalized $\Delta \mathrm{OD}$ decays (at $800 \mathrm{~nm}$ ) of $\mathrm{MK}_{3}-\mathrm{TiO}_{2}$ samples prepared with $0.2 \mathrm{M} \mathrm{Co}^{2+}$, $0.02 \mathrm{M} \mathrm{Co}^{3+}$ in $0.2 \mathrm{M} \mathrm{LiClO}_{4}$ acetonitrile.

the electrolyte concentration was $0.02 \mathrm{M} \mathrm{Co}^{2+}$ and $0.002 \mathrm{M}$ $\mathrm{Co}^{3+}$. Samples employing (Cobpy) $)^{2+/ 3+}$, (Cobpy- $\left.\mathrm{C}_{1}\right)^{2+/ 3+}$, and $(\text { Cobpy-C } 4)^{2+/ 3+}$ showed approximately 10 -fold slower electron transfer kinetics scaling with the 10 times lower redox concentrations. However, samples using $\left(\mathrm{Cobpy}-\mathrm{C}_{9}\right)^{2+/ 3^{+}}$ showed disproportionally faster forward electron transfer by a factor of five compared to the higher concentration case. Electron transfer kinetics is enhanced by thirteen times using a nonyl substituted mediator compared to the methyl substituted one. The results with reduced concentration support that "trapping" of (Cobpy-C 9$)^{2+/ 3+}$ by the hexyl chains on $\mathrm{MK}_{2}$ leading to increased local concentrations is operating. We note that the back electron transfer is dependent on the i) concentration of the oxidized redox mediator at the $\mathrm{TiO}_{2}$ interface, ${ }^{23} \mathrm{ii}$ ) distance of the mediator from the $\mathrm{TiO}_{2}$. The electron lifetime was longer using the nonyl substituted mediator. The trapping of the mediator close to the backbone of the MK2 due to alkyl-alkyl interaction provides a consistent explanation for both faster forward electron transfer and slower back electron transfer. The former is due to better electronic coupling as already explained, while the latter is due to the larger distance from the $\mathrm{TiO}_{2}$ surface as compared to the adsorption of the (Cobpy) ${ }^{3+}$ on the $\mathrm{TiO}_{2}$ surface. We note that in our model, (Cobpy) $)^{3+}$ and $\left(\text { Cobpy- } \mathrm{C}_{1}\right)^{3+}$ ions are predominantly outside of the surface bound molecular layer. When they do diffuse into spaces between the molecules, they can approach the $\mathrm{TiO}_{2}$ surface leading to faster back reaction as compared to (Cobpy- $\left.\mathrm{C}_{1}\right)^{3^{+}}$. The difference is that when the (Cobpy-C9) $)^{3+}$ penetrates the surface-bound molecular layer, it is trapped by the alkyl chain interactions therefore they cannot fully approach the $\mathrm{TiO}_{2}$ surface.

To test the idea of trapping of the redox mediator by the alkyl chains, $\triangle \mathrm{OD}$ decays at reduced $\mathrm{MK}_{2}$ concentrations on the $\mathrm{TiO}_{2}$ surface were measured as shown in Figure 7 . At reduced coverage, the distance between the MK2 molecules should increase, providing easier access by the redox 
mediators to the $\pi$-conjugated backbone as well as increased probability to be close to the $\mathrm{TiO}_{2}$ surface. Both forward and backward electron transfer should be faster compared to the full coverage.

As Figure $\mathbf{7}$ shows, $\triangle \mathrm{OD}$ decays were significantly faster and similar between using the methyl and nonyl substituted mediators at reduced MK2 coverage $\left(\tau_{1 / 2}=2.1 \times 10^{-6}\right.$ and $1.5 \times 10^{-6}$, respectively). The faster forward electron transfer kinetics at lower surface coverage of MK2 are consistent with previous reports by Mori et al. for similarly structured molecules. ${ }^{27}$ The nearly identical forward electron transfer kinetics suggest that the blocking effect between (Cobpy-C9) ${ }^{2+}$ and MK2 was cancelled by increased concentration of (Cobpy-C9) ${ }^{2+}$ near MK2 due to alkyl-alkyl interaction. At reduced coverage, both mediators can access the $\pi$-conjugated segment of the MK2 molecules. Note that the back electron reaction (Figure 3) was generally faster using both mediators at reduced $\mathrm{MK}_{2}$ coverage confirming the higher probability of accessing the $\mathrm{TiO}_{2}$ surface. A noticeably slower back reaction by using the nonyl chain was observed (Figure 3a) as compared to the methyl substituted mediator, which could be explained by the increased distance of the mediator and the $\mathrm{TiO}_{2}$ surface due to trapping by the alkyl chain further away from the $\mathrm{TiO}_{2}$ interface having some effect even at reduced MK2 concentrations.

To test the idea that the interaction is specifically between the alkyl chains both on the MK2 molecules and the mediators, electron transfer between $\mathrm{MK}_{3}$ molecules (without alkyl side chains) and the redox mediators was measured.

Figure 8 shows the $\triangle \mathrm{OD}$ decays recorded with a TA setup with a faster time resolution of up to $0.5 \mathrm{~ns}$. Using (Cobpy- $\left.\mathrm{C}_{1}\right)^{2+/ 3+}$ mediator and $\mathrm{MK}_{3}$ molecule, the forward electron transfer was enhanced by more than an order of magnitude $\left(\tau_{1 / 2}=320 \mathrm{~ns}\right)$ compared to the MK2 molecules. Furthermore, the samples containing the nonyl substituted mediator (Cobpy-C9) ${ }^{2+/ 3+}$ showed five times slower kinetics $(1.3 \mu \mathrm{s})$. This result suggests firstly that both redox mediators can access the $\pi$-conjugated backbone of $\mathrm{MK}_{3}$ more readily compared to the MK2. Secondly, the electron transfer rate is controlled by the length of the alkyl chain on the redox mediator with the longer nonyl chain leading to slower transfer. In the absence of trapping (no alkyl chain on $\mathrm{MK}_{3}$ ), the electronic coupling is reduced between the donor and acceptor by the nonyl chains, leading to slower electron transfer. It is noted based on measurements of molecular coverages of these molecules that $\mathrm{MK}_{3}$ has higher loading on the surface due to its smaller crosssectional area. ${ }^{20}$ However, the free, accessible space between the molecules could be still larger than in the case of $\mathrm{MK}_{2}$, thus electron transfer from the side of the molecule should be easier, see Figure 9. Further supporting this argument of easier access with no alkyl chain on the molecules is the faster back electron transfer in the case of $\mathrm{MK}_{3}-$ adsorbed $\mathrm{TiO}_{2}\left(\mathrm{MK}_{3}-\mathrm{TiO}_{2}\right)$ compared to $\mathrm{MK}_{2}-\mathrm{TiO}_{2}$, both in combination with methyl and nonyl substituted cobalt

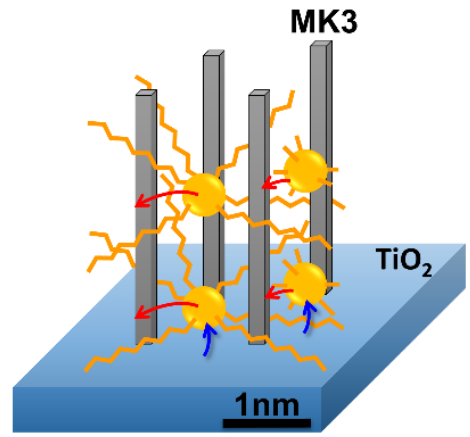

Figure 9. Schematic illustration of electron transfer at the $\mathrm{MK}_{3}-\mathrm{TiO}_{2} /$ electrolyte interface. Blue arrows indicate back electron transfer from the $\mathrm{TiO}_{2}$ to the oxidized mediator, while the red arrows indicate forward electron transfer from the reduced mediator to the oxidized MK2 molecules.

mediators. (Figure $\mathbf{S}_{\mathbf{5}}$ ). The faster back electron transfer suggests easier access to the $\mathrm{TiO}_{2}$ surface, because the mediators can penetrate the $\mathrm{MK}_{3}$ layer more easily. As for the back electron transfer between nonyl versus methyl substituted mediators using $\mathrm{MK}_{3}-\mathrm{TiO}_{2}$, no difference was found. This suggests that the nonyl substituted mediator is not trapped by the $\mathrm{MK}_{3}$ molecules and can access the $\mathrm{TiO}_{2}$ surface to the same extent as the methyl substituted mediator does. In such a case, there is no intrinsic difference in the back reaction rate between the methyl and nonyl substituted mediators. It also follows that the origin of the slower back electron transfer in the case of nonyl substituted mediator and the $\mathrm{MK}_{2}-\mathrm{TiO}_{2}$ (Figure 2a) is not a simple distance dependence dictated by the length or bulkiness of the alkyl chain. It is attributed to the trapping of the mediator away from the $\mathrm{TiO}_{2}$ surface. This explanation raises a further possibility. The flexible linear chain is different from the branched tert-butyl chain in their conformational flexibility. The conformation of the nonyl chain, depending on intermolecular interactions with solvent molecules and the acceptor molecule-adsorbed $\mathrm{TiO}_{2}$ surface, could vary (stretched versus coiled) moderating the distance between the donor and acceptor. Acetonitrile is a polar solvent and the solubility of the nonyl substituted mediator is less than the unsubstituted one (we identified that it more easily crystalized if a small amount of the solvent evaporated). As a next step, repeating the experiments in less-polar electrolytes would be interesting as the difference in interaction between solvent molecules and non-polar alkyl chains on molecules should be less dramatic. In addition to the significance of the findings already mentioned, designing donor / acceptor pairs with built-in flexibility depending on intermolecular interactions is intriguing and may lead to improved designs with tailored back and forward electron transfer rates.

\section{CONCLUSION}


The aim of this study was to clarify what extent long alky chains can block electron transfer between surface bound molecules and redox mediators in solution. Four redox mediators were synthesized including branched tert-butyl and linear nonyl alkyl chain substitutions. As a comparison, methyl substituted mediators having similar redox potentials were prepared. Contrary to expectations, up to thirteen times faster electron transfer was observed when nonyl substituted redox mediators were used in combination with $\mathrm{MK} 2$ molecules decorated by four hexyl chains. These results were explained by intermolecular interactions due to the alkyl chains, leading to the trapping of the redox mediator closer to the $\pi$-conjugated backbone of the MK2 molecules. The trapping increased forward electron transfer rates and slowed down back electron transfer at full $\mathrm{MK}_{2}$ coverage. Using $\mathrm{MK}_{3}-\mathrm{TiO}_{2}$ samples, both forward and back electron transfer was enhanced compared to MK2. However, the forward electron transfer was blocked by the nonyl chain, while no difference in the back reaction was observed. This suggested that the conformation of the nonyl chain may depend on whether the redox mediator approaches the molecules on the surface or the surface of the semiconductor. Such in-built flexibility is an intriguing concept that may allow new designs with large asymmetry between forward and back reaction schemes.

The above results constitute a major departure form current understanding of the effect of molecular structural effects on electron transfer at charge transfer interfaces and has significant consequences for the design of donor / acceptor pairs with controlled electron transfer rates. The results suggest that even a simple modification such as adding insulating alkyl chain is more complex than initially though, and intermolecular interactions between donor acceptor pairs must be more frequently considered.

\section{EXPERIMENTAL SECTION}

Chemicals and materials. MK2 dye (2-cyano-3-[5"'-(9ethyl-9H-carbazol-3-yl)-3',3",3", 4-tetra-n-hexyl-

[2,2'5',2",5",2"']-quarterthiophenyl-5-yl] acrylic acid) (Sigma Aldrich, 95\%), Acetonitrile (Aldrich, 99.999\%), tetrabutylammonium perchlorate (TBAP, >99.0\%, Aldrich), ferrocene (Fc, Aldrich), $\mathrm{TiO}_{2}$ paste (18-NRT, Dyesol), titanium IV chloride $\left(\mathrm{TiCl}_{4}\right.$, Aldrich), Lithium perchlorate $\left(\mathrm{LiClO}_{4}, 95+\%\right.$, Aldrich), fluorine doped tin oxide glass (FTO glass, Hartford Glass Co.), glass microscope slides (1 mm thick, Sail Brand), Surlyn gasket (25 $\mu \mathrm{m}$, DuPont), Titanium diisopropoxide bis(acetylacetonate) (TAA, 75\% in isopropanol, Aldrich), isopropanol (Aldrich), absolute ethanol (Chem-Supply), toluene (Honeywell), sodium hydroxide pellets $(\mathrm{NaOH}$, semiconductor grade, 99.99\% trace metals basis, Aldrich). All cobalt bipyridyl complexes, cobalt (II/III) tris(2,2'-bipyridine), (Cobpy) $)^{2+/ 3+}$, cobalt(II/III) tris (4,4'-dimetyl-2,2'-bipyridine), (Cobpy$\left.\mathrm{C}_{1}\right)^{2+/ 3^{+}}$, cobalt(II/III) tris(4,4'-ditert-butyl-2,2'-bipyridine), $(\text { Cobpy-C } 4)^{2+/ 3+}$, cobalt(II/III) tris 4,4 '-dinonyl-2,2'-bipyri- dine), (Cobpy-C9) $)^{2+/ 3+}$, were synthesized in house as described in Supporting Information. $\mathrm{MK}_{3}$ (2-cyano-3-[5"-(9ethyl-9H-carbazol-3-yl)-[2,2',5',2"] terthiophenyl-5yl]acrylic acid) was synthesized as described in ref. 17 .

Electrochemistry and Driving force calculation. $\mathrm{Cy}$ clic voltammetry $(\mathrm{CV})$ was performed using a potentiostat (65oD, CH Instrument). The reduced forms of the cobalt complexes were dissolved in acetonitrile $(1 \mathrm{mM})$ containing o.1 M TBAP. Before CV measurements, the solutions were purged with dry argon for 10 minutes. A three-electrode setup containing a Pt disk working electrode with a $1.6 \mathrm{~mm}$ diameter, a Pt mesh counter electrode and a o.o1 $\mathrm{M}$ $\mathrm{Ag} / \mathrm{AgNO}_{3}$ reference electrode (MF-2062, BASi) was used. We have measured four identical scans for each redox mediator and repeated the measurement for another independent sample to check reproducibility between consecutive scans and between samples. The redox potential of the $\mathrm{MK}_{2}$ molecules was determined as follows. First, the molecules were adsorbed on $4 \times 4 \mathrm{~mm}$ mesoporous $\mathrm{TiO}_{2}$ films $(2.5 \mu \mathrm{m})$ deposited onto an FTO glass $(2.5 \mathrm{~mm})$ by screen printing. The $\mathrm{TiO}_{2}$ films were sintered at $500{ }^{\circ} \mathrm{C}$ for 30 minutes, then allowed to cool to $110{ }^{\circ} \mathrm{C}$. Then they were immersed into $0.3 \mathrm{mM}$ solutions (toluene for $\mathrm{MK} 2$ ) for 18 hours. A three-electrode system using the MK2 adsorbed $\mathrm{TiO}_{2}$ films as working electrode, a Pt mesh counter electrode and a o.01 $\mathrm{M} \mathrm{Ag} / \mathrm{AgNO}_{3}$ reference electrode were employed for the CV measurements. The supporting electrolyte was o.1 M TBAP in acetonitrile. Trace amount of $\mathrm{Fc}^{+}$ was added to the electrolytes after each the measurement. The CV measurements were repeated to determine the redox potential of $\mathrm{Fc}^{+}$versus the reference electrode.

The formal oxidation potential $\left(\mathrm{E}_{\text {oxi }}\right)$ and reduction potentials $\left(E_{\text {red }}\right)$ were calculated by using the expression $E_{\text {oxi }}$ $=-\left(\mathrm{E}_{\text {oxi }} \mathrm{Vs} . \mathrm{Fc} / \mathrm{Fc}^{+}+5.1\right)(\mathrm{eV})$ and $\mathrm{E}_{\text {red }}=-\left(\mathrm{E}_{\mathrm{red}} \mathrm{Vs} . \mathrm{Fc} / \mathrm{Fc}^{+}+5.1\right)$ $(\mathrm{eV})$, respectively, based on the report by Cardona et al. ${ }^{24}$ The half-wave potential $\left(\mathrm{E}_{1 / 2}\right)$ was determined by equation $E_{1 / 2}=\left(E_{\text {oxi }}+E_{r e d}\right) / 2$. The driving force for forward electron transfer $\left(-\Delta \mathrm{G}_{\mathrm{f}}\right)$ were determined by subtracting the averaged $E_{1 / 2}$ values of cobalt complexes from $E_{1 / 2}$ of $\mathrm{MK} 2 \mathrm{~mol}-$ ecules measured on the $\mathrm{TiO}_{2}$ surface. The driving force for back electron transfer $\left(-\Delta \mathrm{G}_{\mathrm{b}}\right)$ were determined by subtracting the averaged E1/2 values of redox mediators from the assumed conduction band edge level of $\mathrm{TiO}_{2}$ taken from the work done by Kim et al. $3^{\circ}$

Sample fabrication. A TAA solution $(75 \%$ in isopropanol) was diluted with absolute ethanol with the volume ratio of 1:9 of TAA to ethanol). The solution was deposited on FTO glasses kept on a hot plate at $450{ }^{\circ} \mathrm{C}$ by spray pyrolysis. The samples were allowed to cool down to room temperature. The $\mathrm{TiO}_{2}$ paste was deposited by screen printing on the FTO glasses. The FTO / $\mathrm{TiO}_{2}$ electrodes were sintered on a hotplate as follows: $150{ }^{\circ} \mathrm{C}$ for 10 minutes, $325{ }^{\circ} \mathrm{C}$ for 5 minutes, $375{ }^{\circ} \mathrm{C}$ for 5 minutes, $450{ }^{\circ} \mathrm{C}$ for 30 minutes, and $500{ }^{\circ} \mathrm{C}$ for 15 minutes. The films were then immersed into a $0.02 \mathrm{M} \mathrm{TiCl}_{4}$ aqueous solution and kept at $70{ }^{\circ} \mathrm{C}$ for 30 minutes. The surface area of the $\mathrm{TiO}_{2}$ films were $0.64 \mathrm{~cm}^{-2}(8 \times 8 \mathrm{~mm})$ and the thickness of the 
screen printed $\mathrm{TiO}_{2}$ layer was around $3.5 \mu \mathrm{m}$, measured precisely for each sample using a surface profilometer (Dektak 150, Veeco Instruments Inc.). Prior to immersion into the $\mathrm{MK}_{2} / \mathrm{MK}_{3}$ solutions ( $0.3 \mathrm{mM}$, toluene for $\mathrm{MK}_{2}$, DMF for $\mathrm{MK}_{3}$ ), the $\mathrm{TiO}_{2}$ electrodes were re-sintered at 500 ${ }^{\circ} \mathrm{C}$ for 30 minutes and allowed to cool to $110{ }^{\circ} \mathrm{C}$. The immersion time was 18 hours to obtain full coverage. For lower $\mathrm{MK}_{2}$ coverage on $\mathrm{TiO}_{2}$, the $\mathrm{MK} 2$ solutions were diluted to $0.015 \mathrm{mM}$, and the $\mathrm{TiO}_{2}$ films were immersed in the solutions for 2 hours. Platinized counter electrodes were prepared by adding a drop of $10 \mathrm{mM} \mathrm{H}_{2} \mathrm{PtCl}_{6}$ acid solution in ethanol on each of the precut FTO glass substrates, followed by 15 minutes of heating at $400{ }^{\circ} \mathrm{C}$. After the preparation of $\mathrm{MK}_{2} / \mathrm{MK}_{3}-\mathrm{TiO}_{2}$ films, working-counter electrode assemblies were fabricated using $25 \mu \mathrm{m}$ Surlyn gaskets as spacers by heating the sandwich type samples at $120{ }^{\circ} \mathrm{C}$ for 30 seconds. Electrolytes were injected through one of the two pre-drilled holes on the counter electrode by capillary force, followed by sealing with a piece of $25 \mu \mathrm{m}$ Surlyn and glass microscope slides on a hot plate set to 120 ${ }^{\circ} \mathrm{C}$ for 20 seconds.

Stepped light-induced transient measurement of photocurrent and photovoltage (SLIM-PCV) and charge extraction measurement. Stepped light-induced transient measurements of photocurrent and photovoltage and charge extraction measurements were performed as described in the literature. ${ }^{23}$, ${ }^{31}$ A diode laser $(635 \mathrm{~nm}$, Lablaser, Coherent) was employed both for SLIM and charge extraction measurements as the excitation source. The laser light was expanded by a lens to cover the entire active area of the sample. The photovoltage / photocurrent transients were generated by a stepwise change in laser intensity controlled by a function generator, producing $\mathrm{a}<1$ $\mathrm{mV}$ change in photovoltage and a $<10 \%$ change in photocurrent. The current / voltage mode was controlled by an impedance switch. During the charge extraction measurement, the electrical circuit was changed from open to short circuit by a fast switch simultaneously with the laser turn off causing a current transient. Numerical integration of the transient current yielded the amount of charges extracted. Charge density was obtained by dividing the extracted charge amount with the geometric volume of the $\mathrm{TiO}_{2}$ film, not taking into account the porosity of the films. Electron lifetime $(\tau, \mathrm{s})$ was obtained by fitting an exponential function, $\exp (-t / \tau)$, to the voltage decay transient. The diffusion coefficient $\left(\mathrm{D}, \mathrm{cm}^{2} \mathrm{~s}^{-1}\right)$ is calculated from the photocurrent decay, based on $\mathrm{D}=\mathrm{L}^{2} /\left(2.77 \tau_{\mathrm{c}}\right)$, reported by Nakade et al., ${ }^{23}$ where $\mathrm{L}$ is the thickness of the $\mathrm{TiO}_{2}$ layer, and $\tau_{\mathrm{c}}$ is the exponential decay constant.

Transient absorption spectroscopy. To measure forward electron transfer kinetics, transient absorption spectroscopy (TAS) was performed. The same $\mathrm{MK}_{2} / \mathrm{MK}_{3}$ adsorbed $\mathrm{TiO}_{2}$ samples used for SLIM-PCV measurements above were used in TA measurements in transmission mode. Two TA setups were employed depending on the time resolution required for the TA decays.
A microsecond TA setup (10ons to o.o1 s time resolution) employed a Q-switched Nd:YAG laser (INDI-HG QuantaRay, Spectra-Physics) as the pump (532 nm, 6 ns pulses, $10 \mathrm{~Hz}$ repetition rate). A current-controlled quartz halogen lamp (IL1, BENTHAM) was used as a probe light in combination with long pass filters (715 nm, $780 \mathrm{~nm}$, Edmund Optics) and a band pass filter ( $800 \pm 20 \mathrm{~nm}$, Thorlabs) before the sample to avoid photoexcitation by the probe beam. TA decays were measured using a monochromator (CM11o compact 1/8 meter monochromator, Spectral Products) set to $800 \mathrm{~nm}$, a fast photoreceiver (HCA-S-22om-SI, Femto) connected to a voltage amplifier (DHPVA, Femto, AC coupling) and recorded by an oscilloscope (DPO3504, Tektronix). The TA decays were averaged over 512 laser pulse using the average mode of the oscilloscope. The TA decays were determined at three laser intensities adjusted by neutral density filters (Edmund Optics) $(9.5,4.75$, and $2.85 \mu \mathrm{J}$ $\mathrm{cm}^{-2}$ ) confirming no laser intensity dependence of the decays. Charge extraction measurements were also performed under the same experimental conditions as the TA measurements yielding charge densities on the order of $10^{16}$ $\mathrm{cm}^{-3}$.

Some samples $\left(\mathrm{MK}_{3}-\mathrm{TiO}_{2}\right)$ required a faster time resolution as some signal decay was evident on the 100 ns timescale using the slower setup above. For these measurements, a second TA setup with 0.5 ns time resolution was employed. An SBS compressed Nd:YAG laser (SL230, Ekspla) running at $1 \mathrm{~Hz}$ was used as the pump (532 nm, $100 \mathrm{ps}$ pulse width). A pulsed Xe lamp (Xe9oo, Edinburgh Instrument) was used as the probe with a bandpass filter (80o \pm $20 \mathrm{~nm}$, Thorlabs) placed before the sample. The TA signal was recorded at $800 \mathrm{~nm}$ using a monochromator (Oriel Cornerstone $\left.^{\mathrm{TM}}\right)$, a fast photoreceiver $(1601,1 \mathrm{GHz}$, New Focus) and a voltage amplifier (DUPVA-1-6o, Femto), connected to an oscilloscope (DPO5204, Tektronix). $\triangle \mathrm{OD}$ versus time values were merged with the results of the slower TA setup at 100 ns to create one merged file. Several laser pump intensities were measured and no dependence of the decay curves were observed on laser intensity. The reported curves correspond to $4.75 \mu \mathrm{J} \mathrm{cm}^{-2}$ laser intensity.

UV-Visible (UV-Vis) absorption spectroscopy. The absorption spectra of full coverage and reduced coverage $\mathrm{MK}_{2}-\mathrm{TiO}_{2}$ films were recorded by using a Shimadzu UV1800 spectrometer. The full coverage and reduced coverage $\mathrm{TiO}_{2}$ films were immersed into a o.1 $\mathrm{M} \mathrm{NaOH}$ solution (toluene/ethanol $=1 / 1$, volume ratio) for 6 hours to desorb $\mathrm{MK} 2$ molecules from the $\mathrm{TiO}_{2}$ films. Then, the absorption spectra of the solutions were recorded by using PMMA cuvettes with $10 \mathrm{~mm}$ length.

\section{ASSOCIATED CONTENT}

Supporting Information. This Supporting Information is available free of charge via the Internet at http://pubs.acs.org. 
Structure of $\mathrm{D}_{35},^{19}$ synthesis of compounds, cyclic voltammograms of compounds, table of oxidation and reduction potentials, electron diffusion coefficient versus short-circuit current density, electron lifetime versus charge density, open-circuit voltage versus charge density, table containing electron transfer kinetic values, details of data fitting using a stretched exponential function.

\section{AUTHOR INFORMATION}

\section{Corresponding Author}

*attila@uow.edu.au

Notes

The authors declare no competing financial interest.

\section{ACKNOWLEDGMENT}

Funding from the Australian Research Council Centre of Excellence Scheme (Project Number CE 1410oo12) is gratefully acknowledged. The authors would like to thank the Australian National Nanofabrication Facility - Materials node for equipment use.

\section{REFERENCES}

(1) De Vault, D.; Chance, B., Biophys J. 1966, 6, 825-847.

(2) Brunschwig, B. S.; Ehrenson, S.; Sutin, N., J. Am. Chem. Soc. 1984, 106, 6858-6859.

(3) Vlachopoulos, N.; Liska, P.; Augustynski, J.; Grätzel, M., J. Am. Chem. Soc. 1988, 110, 1216-1220.

(4) Bard, A. J.; Fox, M. A., Acc. Chem. Res. 1995, 28, 141-145.

(5) Grätzel, M., nature 2oo1, 414, 338.

(6) Ardo, S.; Meyer, G. J., Chem. Soc. Rev. 2009, 38, 115-164

(7) Hagfeldt, A.; Boschloo, G.; Sun, L.; Kloo, L.; Pettersson, H., Chem. Rev. 2010, 110, 6595-6663.

(8) Robson, K. C.; Hu, K.; Meyer, G. J.; Berlinguette, C. P., J. Am. Chem. Soc. 2013, 135, 1961-1971.

(9) Eberhart, M. S.; Wang, D.; Sampaio, R. N.; Marquard, S. L.; Shan, B.; Brennaman, M. K.; Meyer, G. J.; Dares, C.; Meyer, T. J., J. Am. Chem. Soc. 2017, 139, 16248-16255.

(10) Alibabaei, L.; Brennaman, M. K.; Norris, M. R.; Kalanyan, B.; Song, W.; Losego, M. D.; Concepcion, J. J.; Binstead, R. A.; Parsons, G. N.; Meyer, T. J., Proc. Natl. Acad. Sci. 2013, 110, 20008-20013.

(11) Knauf, R. R.; Kalanyan, B.; Parsons, G. N.; Dempsey, J. L., J. Phys. Chem. C 2015, 119, 28353-2836o.

(12) Wee, K.-R.; Sherman, B. D.; Brennaman, M. K.; Sheridan, M. V.; Nayak, A.; Alibabaei, L.; Meyer, T. J., J. Mater. Chem. A 2016, 4, 2969-2975.

(13) Sapp, S. A.; Elliott, C. M.; Contado, C.; Caramori, S.; Bignozzi, C. A., J. Am. Chem. Soc. 2002, 124, 11215-11222.

(14) Wang, Z.-S.; Koumura, N.; Cui, Y.; Takahashi, M.; Sekiguchi, H.; Mori, A.; Kubo, T.; Furube, A.; Hara, K., Chem. Mater. 2oo8, 20, 3993-4003.

(15) Zhao, L.; Wagner, P.; Barnsley, J. E.; Clarke, T. M.; Gordon, K. C.; Mori S.; Mozer, A. J., Chem. Sci. 2016, 7, 3506-3516.

(16) Rudolf, M.; Kirner, S.; Guldi, D., Chem. Soc. Rev. 2016, 45, 612630.

(17) Koumura, N.; Wang, Z.-S.; Mori, S.; Miyashita, M.; Suzuki, E.; Hara, K., J. Am. Chem. Soc. 20o6, 128, 14256-14257.

(18) Ohta, M.; Koumura, N.; Hara, K.; Mori, S., Electrochem. Commun. 2011, 13, 778-780.

(19) Feldt, S. M.; Gibson, E. A.; Gabrielsson, E.; Sun, L.; Boschloo, G.; Hagfeldt, A., J. Am. Chem. Soc. 2010, 132, 16714-16724.
(20) Ogawa, J.; Koumura, N.; Hara, K.; Mori, S., Jpn. J. Appl. Phys. 2014, 53, 127301.

(21) Feldt, S. M.; Wang, G.; Boschloo, G.; Hagfeldt, A., J. Phys. Chem. C 2011, 115, 21500-21507.

(22) Feldt, S. M.; Lohse, P. W.; Kessler, F.; Nazeeruddin, M. K.; Grätzel, M.; Boschloo, G.; Hagfeldt, A., Phys. Chem. Chem. Phys. 2013, 15, 7087-7097.

(23) Nakade, S.; Kanzaki, T.; Wada, Y.; Yanagida, S., Langmuir 2005, 21, 10803-10807.

(24) Abate, A.; Planells, M.; Hollman, D. J.; Stranks, S. D.; Petrozza, A.; Kandada, A. R. S.; Vaynzof, Y.; Pathak, S. K.; Robertson, N.; Snaith, H. J., Adv. Energy Mater. 2014, 4, 1400166. (25) Nepomnyashchii, A. B.; Parkinson, B., ACS Appl. Mater. Interfaces 2014, 6, 14881-14885.

(26) Cardona, C. M.; Li, W.; Kaifer, A. E.; Stockdale, D.; Bazan, G. C., Adv. Mater. 2011, 23, 2367-2371.

(27) Ogawa, J.-i.; Agrawal, S.; Koumura, N.; Mori, S., J. Phys. Chem. C 2016, 120, 3612-3618.

(28) Anderson, A. Y.; Barnes, P. R.; Durrant, J. R.; O’Regan, B. C., J. Phys. Chem. C 2011, 115, 2439-2447.

(29) Montanari, I.; Nelson, J.; Durrant, J. R., J. Phys. Chem. B 2002, 106, 12203-12210.

(30) Kim, Y. I.; Atherton, S. J.; Brigham E. S.; Mallouk, T. E., J. Phys. Chem. 1993, 97, 11802-11810.

(31) Duffy, N.; Peter, L.; Rajapakse, R.; Wijayantha, K., Electrochem. Commun. 20oo, 2, 658-662. 
Table of Contents

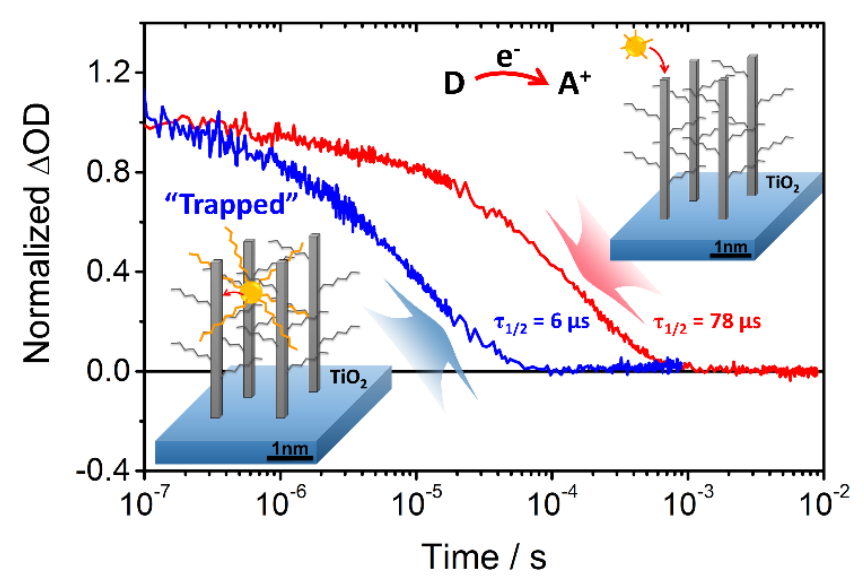

